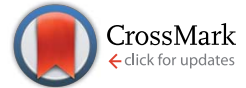

Cite this: RSC Adv., 2017, 7, 10259

Received 10th January 2017 Accepted 31st January 2017

DOI: $10.1039 / \mathrm{c} 7 \mathrm{ra00374a}$

rsc.li/rsc-advances

\section{Surface-plasmon-induced azo coupling reaction between nitro compounds on dendritic silver monitored by surface-enhanced Raman spectroscopy $\dagger$}

\begin{abstract}
Feng-Hsuan Cho, Shan-Chi Kuo and Ying-Huang Lai*
Silver dendrites (Ag-Ds) were prepared by electrodeposition on a glassy carbon electrode (GCE) from a silver(I) sulfate solution containing cysteine. The dendritic Ag surface showed high Raman enhancement, enabling the detection of analytes from dilute solution by surface-enhanced Raman scattering (SERS). 4-Nitrothiophenol (4-NTP) was used as the probe molecule to examine the analyteconcentrating ability and SERS activity of Ag-Ds/GCE composite. The results indicate that the Ag-Ds/ GCE composite exhibits a prominent SERS effect. The lowest 4-NTP detection limit obtained was $5.0 \times$ $10^{-9} \mathrm{M}$. We used a 4-NTP-functionalized Ag-Ds as an analytic and catalytic platform for chemical reactions involving the conversion of $4-$ NTP to $p$ - $p^{\prime}$-dimercaptoazobenzene and azo coupling of 4nitrobenzo-15-crown-5 to 4,4'-mercaptoazobenzene-15-crown-5-ether.
\end{abstract}

\section{Introduction}

Nanometer-sized plasmonic materials (i.e., silver, gold, and composites) exhibit unique chemical and physical properties that may find use in various applications such as molecular sensing, catalysis, and optoelectronics. ${ }^{1-5}$ Surface functionalization of nanostructured materials, which has become an important technique in nanotechnology and surface chemistry, can transform these plasmonic materials into valuable finished products. Plasmon-driven catalytic reactions have attracted attention in recent years because they create a new route for controlling catalytic reactions on metallic substrates. ${ }^{6-10}$ The most studied functional groups of reactants on the surface of metallic substrates exposed to laser excitation are amine or nitro groups dimerized into azo group. For example, 4-aminothiophenol (4-ATP) and 4-nitrothiophenol (4-NTP) dimerize into $p$ - $p^{\prime}$-dimercaptoazobenzene (DMAB). Recent studies suggest that the mechanism of conversion of aromatic nitro compounds is different from that of aromatic amines. For nitro compounds, dimerization is induced by hot electrons produced by surface plasmon decay. ${ }^{9}$ Hot electrons with high kinetic energy transfer to the nitro group of molecules and thus lead to the reduction of nitro-groups to azo-group. On the other hand, the mechanism of conversion of aromatic amines to azo products is independent of hot electrons from surface plasmon decay. Oxidation

Department of Chemistry, Tunghai University, No. 1727, Sec. 4, Taiwan Boulevard, 40704, Taichung, Taiwan. E-mail: yhlai@go.thu.edu.tw; Tel: +886(4)23590121 ext. 32216

$\dagger$ Electronic supplementary information (ESI) available. See DOI: 10.1039/c7ra00374a involves the transfer of plasmonic heat generated at the metal surface to the adsorbed aromatic amines, as well as the participation of oxidizing agents. ${ }^{11}$

Extensive experimental studies of plasmon-driven catalysis have been conducted by using in situ surface-enhanced Raman spectroscopy (SERS). ${ }^{9,12}$ To achieve SERS detection and high efficiency in such plasmonic-driven catalytic reactions, the catalysts should have a large number of active catalytic sites for reaction and sufficient plasmonic cross-sections for light harvesting. Therefore, the vast surface area, catalytic activities, wide-range surface plasmon resonances (SPR), and open threedimensional (3D) nanostructure of metal clusters are key characteristics that we considered for the fabrication of SERSactive substrates for the detection and monitoring of organic analytes during plasmon-driven catalytic reactions..$^{13}$ Although the aforementioned studies examined plasmon-driven dimerization of 4-NTP to DMAB or 4-ATP to DMAB in their SERS experiments, to our knowledge, no study has performed plasmon-driven coupling reactions between different nitro molecules with Ag dendrites (Ag-Ds).

The formation of gold dendrites via electrochemical deposition using cysteine as a blocking molecule has been reported. ${ }^{14,15}$ This enhancement on metal dendrites, which act as very efficient SERS-active sites, may be ascribed to the strong local electromagnetic field generated. ${ }^{16-18}$ Metal dendrites are capable of efficiently catalyzing the transformation of absorbed molecules and sensitively monitoring the structural change of absorbed molecules through SPR derived from metal dendrites on a GCE. In the present study, we used an electrochemical pathway to prepare SERS-active substrates with silver dendrites 
(Ag-Ds). We also investigated the capability of Ag-Ds in sensing a typical organic analyte, 4-NTP, via SERS using laser excitation at $633 \mathrm{~nm}$. Furthermore, we use 4-NTP-functionalized Ag-Ds as an analytic and catalytic platform for chemical reactions involving the conversion of 4-NTP to DMAB and the coupling reaction of 4-nitrobenzo-15-crown-5-ether (4-NB-15-C) to 4,4'mercaptoazobenzene-15-crown-5-ether.

\section{Experimental}

\subsection{Materials}

Silver(I) sulfate ( $\mathrm{Ag}_{2} \mathrm{SO}_{4}$, Merck), 4-NTP, 4-NB-15-C, and cysteine, (Acros, $>99 \%$ ) were used without further purification. Glassy carbon electrode (GCE) plate was the deposition substrate for surface characterization of the electrodeposited Au. Before use, the GCE surface was polished with a nanocloth and alumina slurries $(0.3$ and $0.05 \mu \mathrm{m})$ in sequence. The polished GCE was further cleaned in an ultrasonic bath of water and ethanol for 15 min and was thoroughly rinsed with ultrapure water.

\subsection{Preparation of Ag-Ds}

Ag-Ds were prepared through a modification of Lin's method. ${ }^{\mathbf{1 4}}$ $\mathrm{Ag}$ was electrodeposited on GCE in an aqueous solution of $1.0 \times$ $10^{-3} \mathrm{M} \mathrm{Ag}_{2} \mathrm{SO}_{4}, 0.5 \mathrm{M} \mathrm{H}_{2} \mathrm{SO}_{4}$, and $1.0 \times 10^{-4} \mathrm{M}$ cysteine. A conventional three-electrode system in an electrochemical workstation (CHI 614B) was employed for electrodeposition. A saturated calomel electrode (SCE) served as a reference electrode, and the counter electrode was a platinum wire. Squarewave potential pulses were applied to the GCE at a frequency of $5 \mathrm{~s}^{-1}$ for $1000 \mathrm{~s}$. A pulsed potential form was used to reduce the effect of diffusion limitation on the electrodeposition rate. The lower and higher potentials of the square-wave pulse with respect to the SCE were set at -1.2 and $-0.2 \mathrm{~V}$, respectively.

\subsection{Material characterization}

Field emission scanning electron microscopy (FESEM) measurements were made under a JEOL JSM-6700F microscope operated at an acceleration voltage of $3.0 \mathrm{kV}$. X-ray powder diffraction (XRD) patterns of the Ag-Ds/GCE composite were obtained by using a Philips X'Pert Pro MPD. X-ray photoemission spectroscopy (XPS) measurements were conducted at the 09A1 beamline of the national Synchrotron Radiation Research Center (NSRRC) in Taiwan. The onset of photoemission from an $\mathrm{Au}$ foil attached to the sample holder served as the Fermi level, which corresponds to zero binding energy. The photon energy used to obtain Ag3d XPS spectra was $620 \mathrm{eV}$.

\subsection{SERS measurements}

Raman scattering spectra were obtained by using a microRaman system built in-house. ${ }^{19}$ Samples with $1 \mu \mathrm{m}$ diameter were irradiated with laser at $632.8 \mathrm{~nm}(35 \mathrm{~mW})$ by using a $40 \times 1$ 0.65 N.A. objective lens (Plan N, Olympus). The Raman band of a polystyrene (PS) plate at $1001.4 \mathrm{~cm}^{-1}$ was used to calibrate the spectrometer. The laser exposure time was $0.5 \mathrm{~s}$ for each spectrum. The average of the data for the three spectra was calculated for improved statistics. The SERS samples were prepared by immersing Ag-Ds/GCE composite in ethanol solutions containing different concentrations ( $1 \mathrm{mM}$ to $5 \mathrm{nM}, 1.0 \mathrm{ml}$ ) of 4 NTP for $0.5 \mathrm{~h}$ at ambient temperature. The electrode was then rinsed with deionized water to remove the non-chemisorbed 4NTP.

\section{Results and discussion}

\subsection{Structural characterization of dendritic silver}

3D nanodendritic materials with large surface areas that allow the formation of hot spots are good candidates for increasing sensitivity. ${ }^{20}$ A high density of SERS-active sites at the tips, edges, and junctions of dendrites may facilitate SERS measurement. Many approaches have been developed to prepare dendritic noble metals through galvanic replacement, electrochemical techniques, seeding, and displacement reactions. ${ }^{21-23}$ Electrochemical techniques are conventional, reliable, and robust enough for the preparation of dendrites.

In order to investigate the effect of SERS substrates on surface-plasmon-driven catalytic reactions, Ag-Ds/GCE composites were fabricated by using $0.5 \mathrm{M} \mathrm{H}_{2} \mathrm{SO}_{4}-1.0 \mathrm{mM}$ $\mathrm{Ag}_{2} \mathrm{SO}_{4}-0.10 \mathrm{mM}$ cysteine solution. A stepwise potential from $-0.2 \mathrm{~V}$ to $-1.2 \mathrm{~V}$ for $1000 \mathrm{~s}$ was applied. Fig. 1(a) shows a typical low-magnification SEM image of the prepared Ag-Ds/GCE composite. The SEM images show that the Ag-Ds reached several micrometers in length and had a large rough surface. Ag-Ds under high magnification, Fig. 1(b), had several branches that are oriented radially away from the main stem. There are numerous secondary leaves on each branch, resulting in the formation of Ag-Ds. The angle between each stem and leaf is $c a$. $68^{\circ} \cdot{ }^{14}$ To characterize the composition and structure of the dendrites, XRD measurements were carried out. XRD patterns of the hierarchical Ag-Ds obtained on GCE substrates are shown in Fig. 1(c). Diffraction peaks with sharp features suggest that the prepared Ag-Ds were highly crystalline. Diffraction peaks associated with the (111), (200), (220), (311), and (222) planes of a face-centered cubic (fcc) $\mathrm{Ag}$ structure indicate that the dendritic nanostructures were crystalline silver. ${ }^{24}$ In addition, the intensity ratio of the diffraction peaks is similar to that of diffraction peaks of dendritic crystalline silver. Ratios of the diffraction peak intensities due to the (111) plane relative to those of the (200) and (220) planes were 2.8 and 4.2, respectively. This result indicates that the presence of cysteine in solution induces the electrodeposition of $\mathrm{Ag}$ along the $\langle 111\rangle$ direction of the fcc crystalline structure. ${ }^{25}$ High-resolution XPS measurements were carried out to examine the state of $\mathrm{Ag}$ on the dendrites. Fig. 1(d) shows the Ag3d core-level spectra obtained after electrodeposition of Ag on a GCE. The Ag3d spectra can be fitted with a pair of doublet peaks at 368.0 and $374.0 \mathrm{eV}$, which correspond to $3 \mathrm{~d}_{5 / 2}$ and $3 \mathrm{~d}_{3 / 2}$ levels of metallic $\mathrm{Ag}$ atoms. ${ }^{26}$ All results from SEM, XRD, and XPS measurements suggest that we obtained highly dense and crystalline Ag-Ds.

\subsection{SERS activity of the Ag-Ds/GCE composite}

Dendritic materials with a roughened surface can provide a large surface area and a high density of hot spots for 

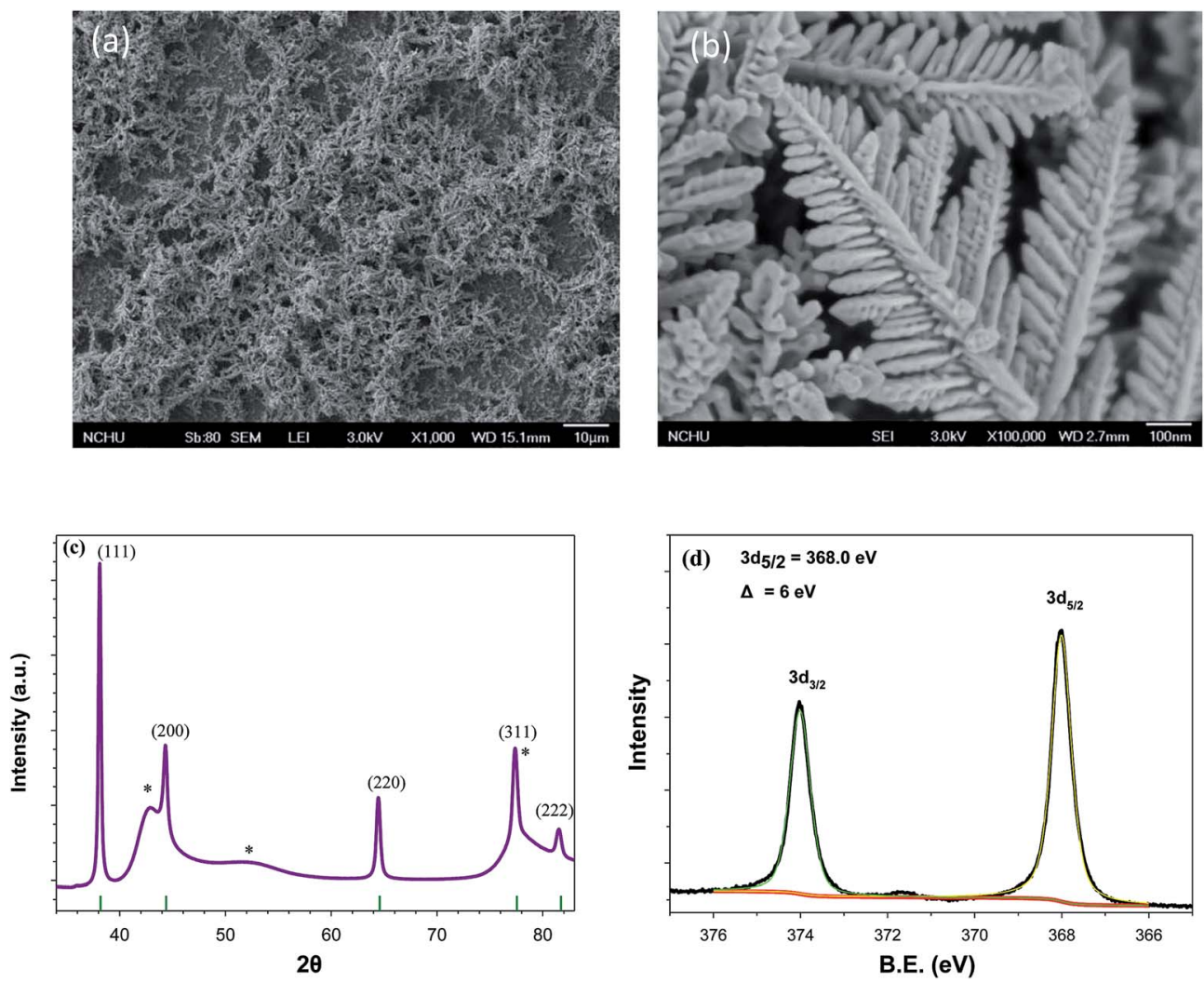

Fig. 1 (a) SEM image of Ag electrodeposited on a GCE at a fixed potential of $-0.8 \mathrm{~V}$ for $1000 \mathrm{~s}$ in a solution of $\mathrm{Ag}_{2} \mathrm{SO}_{4}\left(1.0 \times 10^{-3} \mathrm{M}\right.$ and $\mathrm{H}_{2} \mathrm{SO}_{4}$ $(0.5 \mathrm{M})$ containing cysteine $\left(1.0 \times 10^{-3} \mathrm{M}\right)$. (b) High-magnification SEM image of Ag dendrites. (c) X-ray diffraction patterns of Ag dendrites deposited on GCE for $1000 \mathrm{~s}$. The asterisked peaks are the results for the GCE. (d) XPS spectra of Ag-Ds deposited on GCE. The photo energy used to obtain the spectra was $620 \mathrm{eV}$.

adsorbing reporter molecules. To determine whether the exposed surface of Au-Ds is an effective SERS substrate for the detection of adsorbed reporter molecules, we obtained SERS spectra for Ag-Ds/GCE composite at excitation wavelengths of $633 \mathrm{~nm}$. The dependence of SERS intensities on the 4-NTP concentration was investigated by varying the 4-NTP concentration on the Ag-Ds/GCE composite. Fig. 2(a) shows the SERS spectrum of 4-NTP at $633 \mathrm{~nm}$ laser excitation. It has a distinct, sharp peak even in the case using the $5 \mathrm{nM} 4$-NTP solution. The SERS intensity increased greatly when the 4-NTP concentration increased from $5 \mathrm{nM}$ to $100 \mu \mathrm{M}$. The SERS spectrum of 4-NTP is characterized by major, prominent bands at 1078, 1333, and $1571 \mathrm{~cm}^{-1}$, which respectively correspond to $\mathrm{C}-\mathrm{S}, \mathrm{N}-\mathrm{O}$, and C-C stretching vibrations. ${ }^{19,27,28}$ The SERS results here indicate that when Ag-Ds are densely packed on a GCE, they exhibit a prominent SERS effect. The higher SERS activity of dendritic silver nanostructures was probably caused by their unique morphology and electromagnetic enhancement at sharp edges or/and tips. The strongest band, $\nu\left(\mathrm{NO}_{2}\right)$ is used to identify the presence of 4-NTP and to calculate its intensity. By using the Langmuir adsorption isotherm, models can be used to interpret (1) the trends of Raman intensity versus changing organic molecule concentration, (2) the linear range (detection window) in these dendritic SERS substrates, and (3) the adsorption behavior of reporter molecules. Therefore, SERS intensities of 4-
NTP follow Langmuir's adsorption isotherm equation, as shown in Fig. 2(b):29,30

$$
\theta=\frac{I}{I_{\mathrm{o}}}=\frac{\alpha[4-\mathrm{NTP}]}{1+\alpha[4-\mathrm{NTP}]}
$$

In this equation, $I_{\mathrm{o}}$ is the peak intensity of a saturated 4-NTP monolayer, and $\alpha$ is the adsorption constant of 4-NTP adsorbed on substrates. As the surface coverage $(\theta)$ is related to the 4-NTP concentration, a linear relationship was obtained from $5.0 \times$ $10^{-9}$ to $5.0 \times 10^{-5} \mathrm{M}$. According to the fitted data, the average adsorption constant $(\alpha)$ is $1.44 \times 10^{5}$. That is, a saturated monolayer of 4-NTP adsorbed on the silver surface of the Ag-Ds/ GCE composite when the 4-NTP concentration was higher than $5.0 \times 10^{-5} \mathrm{M}$.

\subsection{Influence of surface coverage of 4-NTP on the dimerization reaction}

Dendritic silver was used as the photocatalytic and analytic platform for plasmon-driven chemical reaction to perform conversions (4-NTP $\rightarrow$ DMAB) with Ag-NPs/GCE composite. A solution containing Ag-Ds/GCE composite and $1.0 \times 10^{-5} \mathrm{M} 4$ NTP $(\theta=0.60)$ was aged for $0.5 \mathrm{~h}$. The electrode was then rinsed with deionized water to remove the non-chemisorbed 4-NTP. In situ SERS spectra of Ag-Ds/GCE composite functionalized with 4-NTP under continuous exposure to a $633 \mathrm{~nm}$ laser with 0.6 



Fig. 2 (a) SERS spectra of the Ag-Ds/GCE composite, after immersion in solutions with the indicated 4-NTP concentrations. The excitation laser wavelength is $633 \mathrm{~nm}$. (b) Adsorption isotherms of 4-NTP on the Ag-Ds/GCE composite obtained according to the $1333 \mathrm{~cm}^{-1} \mathrm{mode}$ in the SERS spectra.

$\mathrm{mW}$ power were then carried obtained. Upon laser exposure for the first $10 \mathrm{~s}$, the SERS spectra exhibited band characteristics of 4-NTP, as shown in Fig. 3. An increase in laser exposure time produced peaks at $1141 \mathrm{~cm}^{-1}$, which correspond to $\beta(\mathrm{C}-\mathrm{H})$; and at 1389 and $1441 \mathrm{~cm}^{-1}$, which correspond to $\nu(\mathrm{N}=\mathrm{N})$. These peaks suggest that a proportion of the 4-NTP molecules were converted to DMAB. ${ }^{11}$ The formation of DMAB may be the result of two adjacent 4-NTP molecules of Ag-Ds/GCE composite interacting with each other, forming DMAB with the aid of surface plasmons of Ag-Ds. Up to $75 \mathrm{~s}$, the intensity of the Raman peak at $1441 \mathrm{~cm}^{-1}$ exceeded that of the peak at 1333 $\mathrm{cm}^{-1}$, but the Raman peaks of $\nu\left(\mathrm{NO}_{2}\right)$ of 4-NTP at 1110 and 1333 $\mathrm{cm}^{-1}$ did not disappear completely, even with further increase

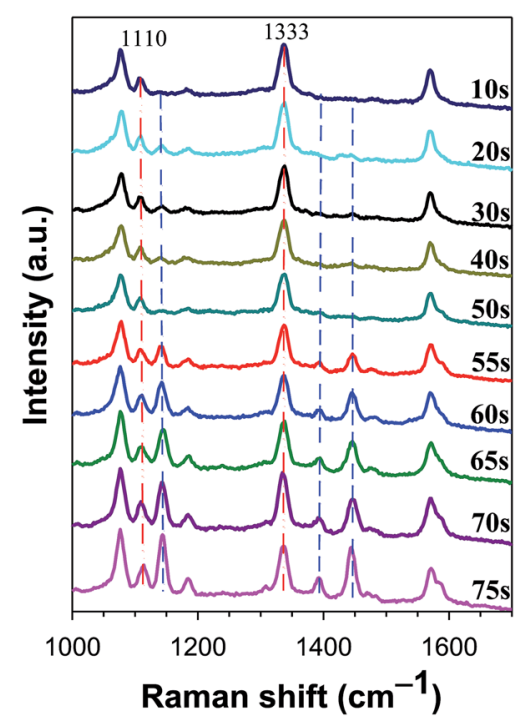

Fig. 3 Time-dependent SERS spectra of 4-NTP $\left(1.0 \times 10^{-5} \mathrm{M}, \theta=\right.$ 0.60 ) on a Ag-Ds/GCE composite under continuous $633 \mathrm{~nm}$ laser excitation. The spectra were obtained by using a laser power of 0.60 $\mathrm{mW}$. of the exposure time. In a separate experiment (Fig. S1 $\dagger$ ), we found that saturated monolayer adsorption of 4-NTP on the Ag surface of the Ag-Ds/GCE composite was completely converted into DMAB. In Fig. $\mathrm{S} 1, \uparrow$ the $\nu\left(\mathrm{NO}_{2}\right)$ of 4 -NTP is difficult to observe. In sharp contrast, the peaks at $1141 \mathrm{~cm}^{-1}$ due to $\beta(\mathrm{C}-$ $\mathrm{H}$ ), as well as those at 1389 and $1441 \mathrm{~cm}^{-1}$ due to $\nu(\mathrm{N}=\mathrm{N})$, are clearly visible in the spectrum. After continuous exposure to $633 \mathrm{~nm}$ laser for $60 \mathrm{~s}$, the 4-NTP molecules were converted to DMAB. $^{12,31}$ Even though the time of laser exposure was extended, the SERS intensity of DMAB showed no obvious change, indicating the completed dimerization of 4-NTP is. In general, SERS effect and chemical reaction rate are increasingly higher with the increase of energy and power. ${ }^{32,33}$ When the wavelength of the laser used was changed from $633 \mathrm{~nm}$ to $532 \mathrm{~nm}$, the rate of dimerization reaction increased. When the $532 \mathrm{~nm}$ excitation laser was used, the time for completely converted into DMAB was several seconds $(<5 \mathrm{~s})$ and shorter than that when the $633 \mathrm{~nm}$ excitation laser was used at the same power. These results suggest that the dense distribution of AgDs on a GCE results in strong enhancement of the local electromagnetic field, making the Ag-Ds/GCE composite an effective SERS platform for detecting structural changes in adsorbed reactants. Furthermore, the substantial difference in the extent of dimerization of 4-NTP could be explained by the surface coverage of 4-NTP.

\subsection{Azo coupling reactions with a 4-NTP-functionalized Ag- Ds/GCE composite}

We attempted to demonstrate the coupling reaction between 4NTP and 4-NB-15-C, as shown in Fig. 4. Both 4-NB-15-C and 4NTP molecules have a $\mathrm{NO}_{2}$ group (Fig. S2 $\dagger$ ), which can be used to study the plasmon-driven azo coupling of different nitro molecules. We modified a Ag-Ds/GCE composite with using surface plasmon to drive chemical reactions and simultaneously recorded SERS spectral changes. As shown in Fig. 5(a), 


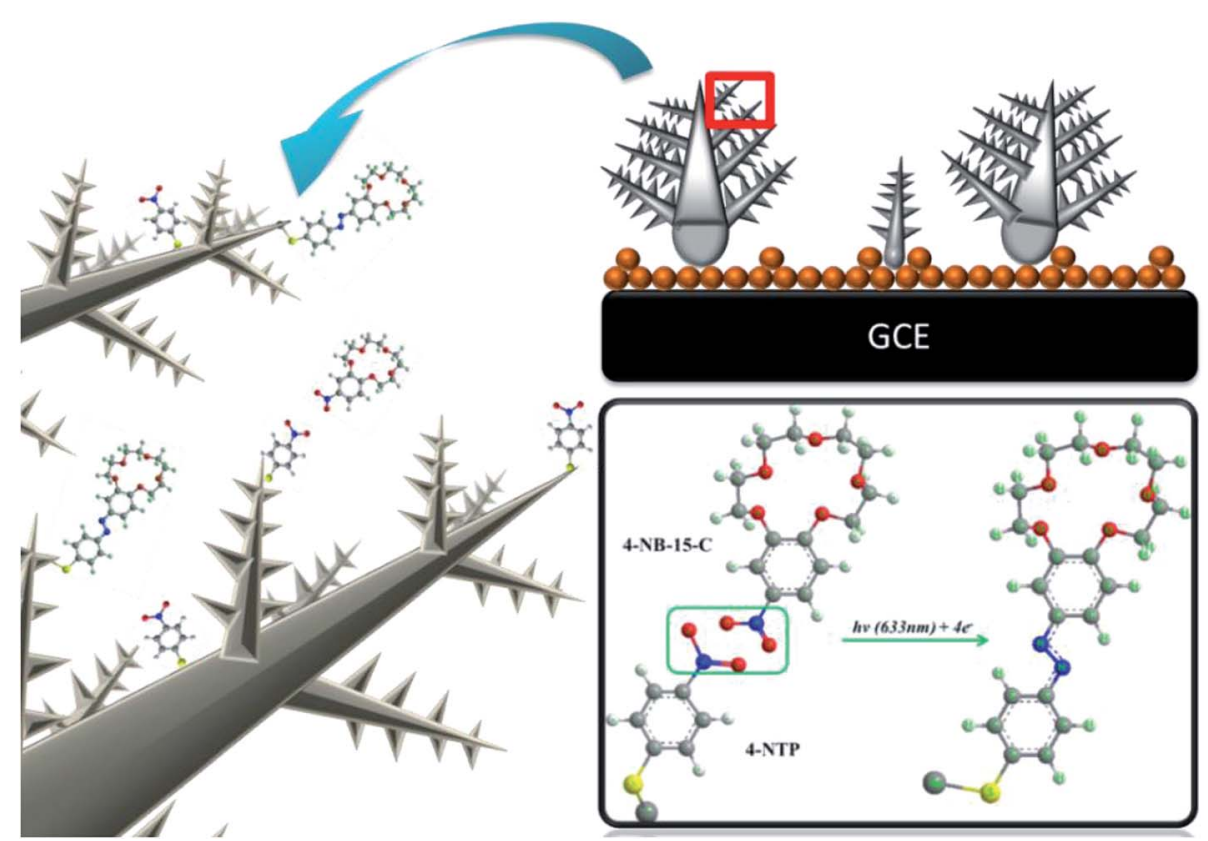

Fig. 4 Schematic illustration of plasmon-driven surface-catalyzed reaction of 4-NB-15-C coupling monitored by using a Ag-Ds/GCE SERS substrate.

4-NTP on the Ag surface of Ag-Ds/GCE composite under continuous exposure to a $633 \mathrm{~nm}$ laser for $300 \mathrm{~s}$ was not converted to $\mathrm{DMAB}$ at low surface coverage of a 0.125 monolayer. The conversion of 4-NTP into DMAB must occur between two or more closely spaced Ag-Ds. At the same surface coverage, when a droplet of the 4-NB-15-C solution was drop cast on a 4-NTPmodified Ag-Ds/GCE composite. As shown in Fig. 5(b), the corresponding time-dependent SERS spectra are similar to the spectrum profile of DMAB. The spectra reveal peaks at 1381 and $1430 \mathrm{~cm}^{-1}$, which correspond to $\nu(\mathrm{N}=\mathrm{N})$, suggesting that a proportion of the 4-NB-15-C molecules coupled with 4-NTP and formed azo compounds. Compared with those of DMAB, the $\mathrm{N}=\mathrm{N}$ vibrations shifted to lower frequencies. Furthermore, the Raman peaks of $\nu\left(\mathrm{NO}_{2}\right)$ of 4 -NTP at $1110 \mathrm{~cm}^{-1}$ diminished significantly, and those of $\nu\left(\mathrm{NO}_{2}\right)$ of $4-\mathrm{NB}-15-\mathrm{C}$ at $1333 \mathrm{~cm}^{-1} \mathrm{did}$ not disappear completely. This observation implies that the limiting reagent in the azo coupling reaction was 4-NTP.

In a controlled experiment, the time-dependent SERS spectra of 4-NB-15-C on a Ag-Ds/GCE composite (Fig. 6) were recorded. We found that no plasmon-driven chemical reaction occurred


Fig. 5 (a) Time-dependent SERS spectra of 4-NTP $\left(1.0 \times 10^{-6} \mathrm{M}, \theta=0.125\right)$ on a Ag-Ds/GCE composite. (b) Time-dependent SERS spectra of 4 NTP $\left(1.0 \times 10^{-6} \mathrm{M}\right)$ on a Ag-Ds/GCE composite under continuous $633 \mathrm{~nm}$ laser excitation in $4-\mathrm{NB}-15-\mathrm{C}$ solution $\left(10 \mu \mathrm{l} 1.0 \times 10^{-5} \mathrm{M}\right)$. The spectra were obtained by using a laser power of $0.60 \mathrm{~mW}$. 


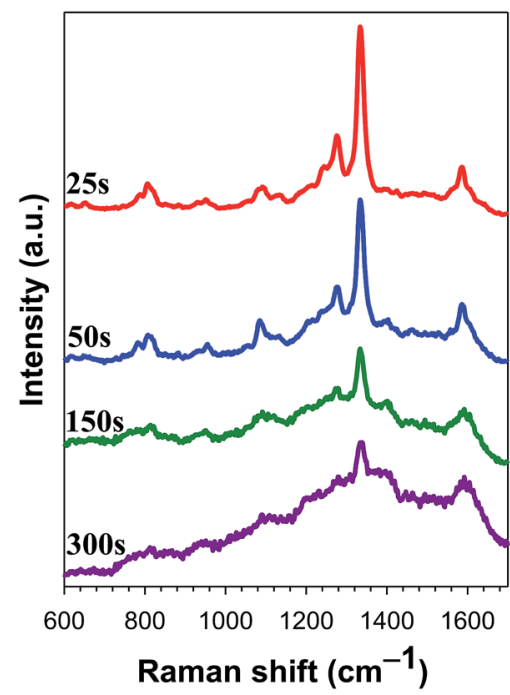

Fig. 6 Time-dependent SERS spectra of 4-NB-15-C on a Ag-Ds/GCE composite.

in the absence of 4-NTP. This result suggests that the formation of azo compounds was driven by the 4-NTP-functionalized AgDs catalyst. Further proof of the foreign 4-NB-15-C molecule coupling with 4-NTP was obtained azo compound with the surface plasmon of Ag-Ds.

\section{Conclusions}

Ag-Ds/GCE assemblies with sharp tips and edges were prepared through a simple electrochemical deposition method. On the basis of comprehensive SERS experiments, we found that GCE is an ideal substrate for Ag-Ds. Dense numbers of Ag-Ds immobilized on the GCE surface enable the concentration of 4-NTP molecules. The dependence of SERS intensities on the concentration of 4-NTP was investigated by varying the 4-NTP concentration of the Ag-Ds/GCE composite. The adsorption of 4NTP onto the Ag surface of the composite occurs through chemisorption, consistent with the Langmuir isotherm. Quantitative SERS detection using the Ag-Ds/GCE composite has detection limits in the $5 \mathrm{nM}$ concentration range. 4-NTP was converted to DMAB on the composite; this finding reveals that the composite is capable of catalyzing the transformation of absorbed molecules and sensitively monitoring the structural change of adsorbed molecules by catalyzing the reduction of AgDs on GCE. Finally, we used 4-NB-15-C molecule, which contains a nitro group, in a reaction observed under a SERS. Further proof of the surface functionalization Au-Ds was obtained by performing a coupling reaction driven by the plasmon-driven catalytic reaction.

\section{Acknowledgements}

The authors gratefully acknowledge the financial support from the Ministry of Science and Technology Taiwan (MOST 1052113-M-029-007).

\section{References}

1 C. Costas, V. López-Puente, G. Bodelón, C. González-Bello, J. Pérez-Juste, I. Pastoriza-Santos and L. M. Liz-Marzán, ACS Nano, 2015, 9, 5567-5576.

2 A. Tripathi, E. D. Emmons, A. W. Fountain, J. A. Guicheteau, M. Moskovits and S. D. Christesen, ACS Nano, 2015, 9, 584593.

3 Y. Wang, B. Yan and L. Chen, Chem. Rev., 2013, 113, 13911428.

4 S. Schlücker, Angew. Chem., Int. Ed., 2014, 53, 4756-4795.

5 J. A. Dieringer, K. L. Wustholz, D. J. Masiello, J. P. Camden, S. L. Kleinman, G. C. Schatz and R. P. Van Duyne, J. Am. Chem. Soc., 2009, 131, 849-854.

6 M. Sun, Z. Zhang, H. Zheng and H. Xu, Sci. Rep., 2012, 2, 647.

7 X. Liang, T. You, D. Liu, X. Lang, E. Tan, J. Shi, P. Yin and L. Guo, Phys. Chem. Chem. Phys., 2015, 17, 10176-10181.

8 P. Li, B. Ma, L. Yang and J. Liu, Chem. Commun., 2015, 51, 11394-11397.

9 L. Kang, J. Chu, H. Zhao, P. Xu and M. Sun, J. Mater. Chem. C, 2015, 3, 9024-9037.

10 L. Cui, P. Wang, Y. Fang, Y. Li and M. Sun, Sci. Rep., 2015, 5, 11920.

11 P. Xu, L. Kang, N. H. Mack, K. S. Schanze, X. Han and H.-L. Wang, Sci. Rep., 2013, 3, 2997.

12 H.-Y. Wu, Y.-H. Lai, M.-S. Hsieh, S.-D. Lin, Y.-C. Li and T.-W. Lin, Adv. Mater. Interfaces, 2014, 1, 1400119.

13 H. Liu, Z. Yang, L. Meng, Y. Sun, J. Wang, L. Yang, J. Liu and Z. Tian, J. Am. Chem. Soc., 2014, 136, 5332-5341.

14 T.-H. Lin, C.-W. Lin, H.-H. Liu, J.-T. Sheu and W.-H. Hung, Chem. Commun., 2011, 47, 2044-2046.

15 F.-H. Cho, Y.-C. Lin and Y.-H. Lai, Appl. Surf. Sci., 2017, 402, 147-153.

16 Q. Teng, Z. Yongjin, L. Jiaqi, Z. Wenjun, L. Xianzhong, C. Tiejun and K. C. Paul, J. Phys. D: Appl. Phys., 2009, 42, 175403.

17 K. Jasuja and V. Berry, ACS Nano, 2009, 3, 2358-2366.

18 X. Tang, W. Cai, L. Yang and J. Liu, Nanoscale, 2013, 5, 11193-11199.

19 L.-P. Wang, S.-C. Kuo, U. S. Jeng and Y.-H. Lai, RSC Adv., 2015, 5, 37323-37329.

20 G. Lu, C. Li and G. Shi, Chem. Mater., 2007, 19, 3433-3440.

21 X. Bai, Y. Gao and L. Zheng, CrystEngComm, 2011, 13, 35623568.

22 S. J. Lee, A. R. Morrill and M. Moskovits, J. Am. Chem. Soc., 2006, 128, 2200-2201.

23 H. M. Song, L. Deng and N. M. Khashab, Nanoscale, 2013, 5, 4321-4329.

24 G. Zhang, S. Sun, M. Cai, Y. Zhang, R. Li and X. Sun, Sci. Rep., 2013, 3, 1526.

25 M. Tian, J. Wang, J. Kurtz, T. E. Mallouk and M. H. W. Chan, Nano Lett., 2003, 3, 919-923.

26 Y. Wu, Z. Wang, S. Chen, J. Wu, X. Guo and Z. Liu, RSC Adv., 2015, 5, 87151-87156.

27 E. M. van Schrojenstein Lantman, T. Deckert-Gaudig, A. J. G. Mank, V. Deckert and B. M. Weckhuysen, Nat. Nanotechnol., 2012, 7, 583-586. 
28 S.-C. Kuo, J.-J. Tasi, J.-S. Li, Z.-H. Hou, C.-H. Li, U.-S. Jeng and Y.-H. Lai, APL Mater., 2014, 2, 113310.

29 H. Wang, C. S. Levin and N. J. Halas, J. Am. Chem. Soc., 2005, 127, 14992-14993.

30 Y.-H. Lai, S.-C. Kuo, Y.-C. Hsieh, Y.-C. Tai, W.-H. Hung and U. S. Jeng, RSC Adv., 2016, 6, 13185-13192.
31 X. Zhang, P. Wang, Z. Zhang, Y. Fang and M. Sun, Sci. Rep., 2014, 4, 5407.

32 R. A. Álvarez-Puebla, J. Phys. Chem. Lett., 2012, 3, 857-866. 33 T.-T. Tasi, T.-W. Lin, L.-D. Shao and H.-H. Shen, RSC Adv., 2016, 6, 29453-29459. 